\title{
Size estimates of unknown boundaries with a Robin-type condition
}

\author{
Michele Di Cristo
}

Politecnico di Milano, Dipartimento di Matematica, Piazza Leonardo da Vinci 32, 20133 Milano, Italy

(michele.dicristo@polimi.it)

\section{Eva Sincich}

Università degli Studi di Trieste, Dipartimento di Matematica e Geoscienze, via Valerio 12/1, 34127 Trieste, Italy (esincich@units.it)

\section{Sergio Vessella}

Università di Firenze,

Dipartimento di Matematica e Informatica 'Ulisse Dini', Viale Morgagni, 67/a 50134 Firenze, Italy

(sergio.vessella@dmd.unifi.it)

(MS received 28 March 2014; accepted 11 January 2016)

\author{
We deal with the problem of determining an unknown part of the boundary of an \\ electrical conductor that is inaccessible for external observation and where a \\ corrosion process is going on. We obtain estimates of the size of this damaged region \\ from above and below. \\ Keywords: inverse problems; size estimates; boundary determination \\ 2010 Mathematics subject classification: Primary 35J58; 35R30
}

\section{Introduction}

We consider an electrical conductor $\Omega$ whose boundary is not fully observable, and denote by $\Gamma$ the portion of $\partial \Omega$ where it is possible to make measurements. The aim of this paper is to extract information on an unknown subset $E$ contained in $\partial \Omega \backslash$ $\Gamma$, where a corrosion process is going on, by performing boundary measurements on $\Gamma$. These problems arise in non-destructive testing of materials and modelling phenomena of surface corrosion in metals (see $[16,23]$ ).

Prescribing a current density $g$ supported on $\Gamma$ such that $g=0$ on $\partial \Omega \backslash \Gamma$, we induce a potential $u$ solution to the problem

$$
\left.\begin{array}{rl}
\Delta u=0 & \text { in } \Omega, \\
\frac{\partial u}{\partial \nu}+\gamma u=g & \text { on } \partial \Omega,
\end{array}\right\}
$$


where $\gamma$ denotes the surface impedance in the form

$$
\gamma(x)=\gamma_{0}(x) \chi_{\Gamma}+k \chi_{E} \quad \text { for any } x \in \partial \Omega,
$$

where $k$ is a constant whose value is unknown and $\gamma_{0} \equiv 0$ in $\partial \Omega \backslash \Gamma$. The case in which $k$ is replaced by a variable function can be treated similarly with minor adjustments, while on the remaining portion of the boundary $\partial \Omega \backslash E$ the impedance term $\gamma$ is fully known.

Our goal is to bound the measure of $E$ by comparing the solution $u$ on the boundary with the solution $u_{0}$ of the 'unperturbed' problem

$$
\left.\begin{array}{rl}
\Delta u_{0}=0 & \text { in } \Omega, \\
\frac{\partial u_{0}}{\partial \nu}+\gamma_{0} u_{0}=g & \text { on } \partial \Omega,
\end{array}\right\}
$$

where $E=\emptyset$, i.e. is, in principle, completely known. Note that $\partial u_{0} / \partial \nu$ vanishes outside $\Gamma$.

Specifically, using similar arguments to those developed in the context of the inverse inclusion problem (see [5] and the references therein), we deduce information on the size of $E$ by analysing the so-called power gap, defined as

$$
W-W_{0}=\int_{\partial \Omega} g u \mathrm{~d} \sigma-\int_{\partial \Omega} g u_{0} \mathrm{~d} \sigma=\int_{\Gamma} g\left(u-u_{0}\right) \mathrm{d} \sigma .
$$

Note that the quantities $W$ and $W_{0}$ can be computed from the boundary data that we measure and are meaningful from a physical viewpoint as they represent the power required to maintain the boundary current $g$.

The idea of bounding the size of an unknown object $D$ enclosed in a given domain $\Omega$ goes back to Friedman [13]. The key point is to extract as much information as possible from the boundary measurements available. More precisely, the approach we follow is that proposed by Alessandrini and Rosset [3] and Kang et al. [15] and subsequently refined by Alessandrini et al. [4].

The basic aim is to gain information on the hidden boundary by studying the power gap, which is sensitive to the presence of the defect. In particular, since such a power gap contains information at the accessible boundary, it is possible to extend this to the inaccessible part of the boundary in a quantitative manner and thus obtain information on its size. This procedure follows the lines of similar problems studied in $[3,15]$ and later developed in $[5,8,10-12,19,20]$. The main novelty of this paper relies on the evaluation of a defect located on the boundary. Such a new feature requires an original approach to relate the power gap and the size of the defect. In order to overcome such a difficulty we find it convenient to analyse the problem in an abstract Hilbert setting (see $\S 3$ ). Due to its general character, this argument can be applied to inverse problems in other practical contexts. The main technical arguments are based on the use of the three-spheres inequality and the doubling inequality at the boundary as unique continuation tools that allow us to extract information on the unknown defect from the interior and the boundary values of the solution. Another issue that arises in dealing with boundary defects concerns the use of quantitative estimates. With the introduction of a suitable norm (see remark 2.3) and quantitative estimates of unique continuation 
(see proposition 4.2), it is possible to obtain the desired bounds on the size of the corroded part.

The plan of the paper is the following. In $\S 2$ we define our notation and state the main theorem. In $\S 3$ we present an abstract formulation of our problem that will be applied in $\S 4$ to prove our main result.

\section{Assumptions and main result}

For a given vector $x=\left(x_{1}, x_{2}, \ldots, x_{n}\right)$ in $\mathbb{R}^{n}$, we write $x=\left(x^{\prime}, x_{n}\right)$, where $x^{\prime}=$ $\left(x_{1}, \ldots, x_{n-1}\right)$. Moreover, we denote by $B_{r}(x)$ and $B_{r}^{\prime}(x)$ the open balls of radius $r$ centred at $x$ and $x^{\prime}$ in $\mathbb{R}^{n}, \mathbb{R}^{n-1}$, respectively.

Definition 2.1. Let $\Omega$ be a bounded domain in $\mathbb{R}^{n}$. Given $k$, $\alpha$ with $k \in \mathbb{N}$, $0<\alpha \leqslant 1$, we say that a portion $S$ of $\partial \Omega$ is of class $C^{k, \alpha}$ with constants $r_{0}, M$ if, for any $P \in S$, there exists a rigid transformation of coordinates under which we have $P=0$ and

$$
\Omega \cap B_{r_{0}}(0)=\left\{x \in B_{r_{0}}(0): x_{n}>\psi\left(x^{\prime}\right)\right\},
$$

where $\psi$ is a $C^{k, \alpha}$ function on $B_{r_{0}}^{\prime}(0)$ satisfying

$$
\begin{gathered}
\psi(0)=0, \\
\nabla \psi(0)=0 \quad \text { when } k \geqslant 1, \\
\|\psi\|_{C^{k, \alpha}\left(B_{r_{0}}^{\prime}(0)\right)} \leqslant M r_{0} .
\end{gathered}
$$

When $k=0$ and $\alpha=1$, we also say that $S$ is of Lipschitz class with constants $r_{0}$ and $M$.

REMARK 2.2. We have chosen to normalize all norms in such a way that their terms are dimensionally homogeneous and coincide with the standard definition as the dimensional parameter equals 1. For instance, the meaning of the norm appearing in the previous definition is as follows:

$$
\|\psi\|_{C^{k, \alpha}\left(B_{r_{0}}^{\prime}(0)\right)}=\sum_{i=0}^{k} r_{0}^{i}\left\|D^{i} \psi\right\|_{L^{\infty}\left(B_{r_{0}}^{\prime}(0)\right)}+r_{0}^{k+\alpha}\left|D^{k} \psi\right|_{\alpha, B_{r_{0}}^{\prime}(0)},
$$

where

$$
\left|D^{k} \psi\right|_{\alpha, B_{r_{0}}^{\prime}(0)}=\sup _{x^{\prime}, y^{\prime} \in B_{r_{0}}^{\prime}, x^{\prime} \neq y^{\prime}} \frac{\left|D^{k} \psi\left(x^{\prime}\right)-D^{k} \psi\left(y^{\prime}\right)\right|}{\left|x^{\prime}-y^{\prime}\right|^{\alpha}} .
$$

Similarly, we shall set

$$
\begin{aligned}
\|u\|_{L^{2}(\Omega)} & =r_{0}^{-n / 2}\left(\int_{\Omega} u^{2}\right)^{1 / 2} \\
\|u\|_{H^{1}(\Omega)} & =r_{0}^{-n / 2}\left(\int_{\Omega} u^{2}+r_{0}^{2} \int_{\Omega}|\nabla u|^{2}\right)^{1 / 2} .
\end{aligned}
$$

Let $\langle\cdot, \cdot\rangle_{H^{-1 / 2}, H^{1 / 2}}$ denote the duality pairing between $H^{-1 / 2}(\partial \Omega)$ and $H^{1 / 2}(\partial \Omega)$ based on the $L^{2}$ scalar product. Given the open and connected portion $\Gamma$ of $\partial \Omega$, 
we introduce the trace space $H_{00}^{1 / 2}(\Gamma)$ as the interpolation space $\left[H_{0}^{1}(\Gamma), L^{2}(\Gamma)\right]_{1 / 2}$ (see $[17$, ch. 1]). Let us now consider the following space of distributions:

$$
H^{-1 / 2}(\Gamma)=\left\{\eta \in H^{-1 / 2}(\partial \Omega) \mid\langle\eta, \varphi\rangle=0 \forall \varphi \in H_{00}^{1 / 2}(\partial \Omega \backslash \bar{\Gamma})\right\} .
$$

\subsection{Assumptions on the domain $\Omega$}

Given constants $r_{0}, M>0$, we assume that $\Omega \subset \mathbb{R}^{n}, n \geqslant 2$, and

$\Omega$ is of Lipschitz class with constants $r_{0}, M$.

Furthermore, given $L>0$, we assume that

$$
|\partial \Omega| \leqslant L r_{0}^{n-1} .
$$

In addition, we assume that the portion of the boundary

$$
\partial \Omega \backslash \Gamma \text { is of class } C^{1,1} \text { with constants } r_{0}, M \text {. }
$$

\subsection{Assumptions on the surface impedance $\gamma$}

Given an open and connected subset $E$ of $\partial \Gamma \backslash \bar{\Gamma}$ and an open and connected subset $\Gamma_{0}$ of $\Gamma$, we assume that

$$
\gamma \in L^{\infty}(\partial \Omega)
$$

Moreover, for a given constant $c_{0}, 0<c_{0} \leqslant 1$, we have that

$$
\gamma(x) \geqslant \frac{c_{0}}{r_{0}}>0 \quad \text { on } \Gamma_{0} .
$$

Finally, for a given function $\gamma_{0}(x) \in L^{\infty}(\partial \Omega)$ supported on $\Gamma$ and such that

$$
\gamma_{0}(x) \leqslant c_{0}^{-1} / r_{0}
$$

we have that

$$
\gamma(x)=\gamma_{0}(x) \chi_{\Gamma}+k \chi_{E},
$$

where $k>0$ is an unknown constant such that

$$
0<\bar{k}_{0}<k r_{0}<\bar{k}_{1}
$$

for given constants $\bar{k}_{0}$ and $\bar{k}_{1}$.

Here and in the following we shall set

$$
\begin{aligned}
\gamma(x) & =\frac{\bar{\gamma}(x)}{r_{0}}, \\
\gamma_{0}(x) & =\frac{\overline{\gamma_{0}}(x)}{r_{0}}, \\
k & =\frac{\bar{k}}{r_{0}} .
\end{aligned}
$$




\subsection{Assumptions on the given data $g$}

Given $g_{0}>0$ we assume that

$$
\|g\|_{H^{-1 / 2}(\Gamma)} \leqslant g_{0} .
$$

Furthermore, given $F>0$ we assume that

$$
\frac{\|g\|_{H^{-1 / 2}(\Gamma)}}{\|g\|_{H^{-1}(\Gamma)}} \leqslant F .
$$

This ratio (called frequency) takes into account the oscillatory character of the boundary data. Other choices of norm are possible and we refer the reader to [5] for a discussion on this topic.

REMARK 2.3. We first observe that the standard norm in $H^{1}(\Omega)$ and the norm

$$
\|u\|_{*}=r_{0}^{-n / 2}\left(r_{0}^{2} \int_{\Omega}|\nabla u|^{2} \mathrm{~d} x+r_{0} \int_{\Gamma_{0}} u^{2} \mathrm{~d} \sigma\right)^{1 / 2},
$$

are equivalent.

Indeed, we note that, on the one hand, by the standard trace estimate we have

$$
\|u\|_{H^{1 / 2}\left(\Gamma_{0}\right)} \leqslant C\|u\|_{H^{1}(\Omega)},
$$

where $C>0$ is a constant depending only on $L$ and $M$. The above inequality leads to

$$
r_{0}^{2} \int_{\Omega}|\nabla u|^{2} \mathrm{~d} x+r_{0} \int_{\Gamma_{0}} u^{2} \mathrm{~d} \sigma \leqslant C\left(r_{0}^{2} \int_{\Omega}|\nabla u|^{2} \mathrm{~d} x+\int_{\Omega} u^{2} \mathrm{~d} x\right),
$$

where $C>0$ is a constant depending only on $L$ and $M$.

On the other hand, by the argument in [6, example 3.6], we deduce that

$$
r_{0}^{2} \int_{\Omega}|\nabla u|^{2} \mathrm{~d} x+\int_{\Omega} u^{2} \mathrm{~d} x \leqslant C\left(r_{0}^{2} \int_{\Omega}|\nabla u|^{2} \mathrm{~d} x+r_{0} \int_{\Gamma_{0}} u^{2} \mathrm{~d} \sigma\right),
$$

where $C>0$ is a constant depending only on $L$ and $M$.

Again denoting by $\langle\cdot, \cdot\rangle_{H^{-1 / 2}, H^{1 / 2}}$ the duality pairing between $H^{-1 / 2}(\partial \Omega)$ and $H^{1 / 2}(\partial \Omega)$, with a slight abuse of notation, we shall write

$$
\langle g, f\rangle_{H^{-1 / 2}, H^{1 / 2}}=\int_{\partial \Omega} g f \mathrm{~d} \sigma
$$

for any $g \in H^{-1 / 2}(\partial \Omega)$ and $f \in H^{1 / 2}(\partial \Omega)$.

REMARK 2.4. By solution to (1.1) we mean a function $u \in H^{1}(\Omega)$ such that

$$
\int_{\Omega} \nabla u \cdot \nabla v \mathrm{~d} x+\int_{\partial \Omega} \gamma(x) u v \mathrm{~d} \sigma=\int_{\partial \Omega} g v \mathrm{~d} \sigma \quad \forall v \in H^{1}(\Omega) .
$$

As a consequence of remark 2.3, we deduce that the existence and uniqueness of the weak solution to problem (1.1) follow from standard theory on the boundary-value problem for the Laplace equation and the sign condition (2.7). 
The inverse problem we are addressing is to estimate the size of the corroded part $E$ of the boundary from a knowledge of Cauchy data $\left\{g,\left.u\right|_{\Gamma}\right\}$. To do this we shall compare $u$ with the solution $u_{0}$ of the problem when $E=\emptyset$ and $\gamma \equiv \gamma_{0}$. Precisely, $u_{0} \in H^{1}(\Omega)$ is such that

$$
\int_{\Omega} \nabla u_{0} \cdot \nabla v \mathrm{~d} x+\int_{\partial \Omega} \gamma_{0}(x) u_{0} v \mathrm{~d} \sigma=\int_{\partial \Omega} g v \mathrm{~d} \sigma \quad \forall v \in H^{1}(\Omega) .
$$

As earlier, we denote by $W$ and $W_{0}$ the power required to maintain the current density $g$ on $\partial \Omega$ when $E$ is and is not present, respectively, namely

$$
\begin{gathered}
W=\int_{\partial \Omega} g u \mathrm{~d} \sigma=\int_{\Omega} \nabla u \cdot \nabla u \mathrm{~d} x+\int_{\partial \Omega} \gamma u^{2} \mathrm{~d} \sigma, \\
W_{0}=\int_{\partial \Omega} g u_{0} \mathrm{~d} \sigma=\int_{\Omega} \nabla u_{0} \cdot \nabla u_{0} \mathrm{~d} x+\int_{\partial \Omega} \gamma_{0} u_{0}^{2} \mathrm{~d} \sigma .
\end{gathered}
$$

From now on we shall refer to the following set of quantities as the a priori data: $M, L, \bar{k}_{0}, \bar{k}_{1}, c_{0}, g_{0}, F$.

We can now state the main result we want to prove.

TheOREM 2.5. Let $\Omega \subset \mathbb{R}^{n}$ be a bounded domain whose boundary is of class $C^{0,1}$. Let $\gamma, \gamma_{0} \in L^{\infty}(\partial \Omega)$ defined as above. Then there exist positive constants $C_{1}, C_{2}, p>$ 1 depending only on the a priori data such that

$$
C_{1} r_{0}{ }^{n-1} \frac{W-W_{0}}{W_{0}} \leqslant|E| \leqslant C_{2} r_{0}{ }^{n-1}\left(\frac{W-W_{0}}{W_{0}}\right)^{1 / p} .
$$

\section{Abstract formulation}

To prove theorem 2.5 we shall make use of techniques developed in the context of the inverse conductivity problem [5]. The difference from other situations is that we want to determine a defect in the external boundary of the specimen, whereas in the other cases the inhomogeneity is fully contained in the domain. To overcome this difficulty we shall rephrase our argument in an abstract way, disconnecting it from the physical context.

We denote by $H$ a Hilbert space and by $H^{\prime}$ its dual. Let $a_{1}(\cdot, \cdot)$ and $a_{0}(\cdot, \cdot)$ be two bilinear symmetric forms on $H$ and let $F \in H^{\prime}$. By the Lax-Milgram theorem, there exist $u_{1}$ and $u_{0}$ in $H$ such that

$$
a_{j}\left(u_{j}, v\right)=\langle F, v\rangle \quad \forall v \in H, j=0,1,
$$

where $\langle\cdot, \cdot\rangle$ denotes the duality pairing between $H$ and $H^{\prime}$.

Lemma 3.1. The following inequalities hold:

$$
\begin{aligned}
& J_{1}:=a_{0}\left(u_{1}-u_{0}, u_{1}-u_{0}\right)-\left[a_{1}\left(u_{0}, u_{0}\right)-a_{0}\left(u_{0}, u_{0}\right)\right]=\left\langle F, u_{1}-u_{0}\right\rangle, \\
& J_{2}:=a_{0}\left(u_{0}-u_{1}, u_{0}-u_{1}\right)-\left[a_{0}\left(u_{1}, u_{1}\right)-a_{1}\left(u_{1}, u_{1}\right)\right]=-\left\langle F, u_{1}-u_{0}\right\rangle, \\
& J_{3}:=a_{0}\left(u_{1}, u_{0}\right)-a_{1}\left(u_{1}, u_{0}\right)=\left\langle F, u_{1}-u_{0}\right\rangle .
\end{aligned}
$$


Proof. Let us verify $(3.1 a)$.

$$
\begin{aligned}
a_{0}\left(u_{1}-u_{0}, u_{1}-\right. & \left.u_{0}\right)-\left[a_{1}\left(u_{0}, u_{0}\right)-a_{0}\left(u_{0}, u_{0}\right)\right] \\
& =a_{1}\left(u_{1}, u_{1}\right)-2 a_{1}\left(u_{1}, u_{0}\right)+a_{1}\left(u_{0}, u_{0}\right)-a_{1}\left(u_{0}, u_{0}\right)+a_{0}\left(u_{0}, u_{0}\right) \\
& =\left\langle F, u_{1}\right\rangle-2\left\langle F, u_{0}\right\rangle+\left\langle F, u_{0}\right\rangle=\left\langle F, u_{1}-u_{0}\right\rangle .
\end{aligned}
$$

Equalities $(3.1 b)$ and $(3.1 c)$ can be obtained similarly.

We now define

$$
G(u):=a_{1}(u, u)-a_{0}(u, u), \quad u \in H .
$$

Let us observe that $G$ is a functional depending on the defect. We also define

$$
\alpha(u, v):=\frac{1}{4}[G(u+v)-G(u-v)], \quad u, v \in H .
$$

Trivially, we have

$$
a_{1}(u, v)=a_{0}(u, v)+\alpha(u, v), \quad u, v \in H .
$$

Lemma 3.2. If, for every $u \in H$, either $\alpha(u, u) \geqslant 0$ or $\alpha(u, u) \leqslant 0$, then

$$
|\alpha(u, v)| \leqslant|\alpha(u, u)|^{1 / 2}|\alpha(v, v)|^{1 / 2}
$$

for every $u, v \in H$.

Proof. If $\alpha(u, u)=0$ and $\alpha(v, v)=0$, then, assuming $\alpha(w, w) \geqslant 0$ for every $w \in H$, we would have

$$
0 \leqslant \alpha(u+t v, u+t v)=2 t \alpha(u, v) \quad \forall t \in \mathbb{R},
$$

which implies $\alpha(u, v)=0$ and (3.2) is proved.

If $\alpha(u, u) \neq 0$ or $\alpha(v, v) \neq 0$, then assuming, for instance, $\alpha(v, v)>0$, we would have

$$
0 \leqslant \alpha(u+t v, u+t v)=t^{2} \alpha(v, v)+2 t \alpha(u, v)+\alpha(u, u) \quad \forall t \in \mathbb{R},
$$

from which

$$
(\alpha(u, v))^{2}-\alpha(u, u) \alpha(v, v) \leqslant 0
$$

and (3.2) follows.

If $\alpha(w, w) \leqslant 0$ for every $w \in H$, the thesis follows by similarly applying the above argument to $-\alpha(\cdot, \cdot)$.

Defining

$$
\delta W=\left\langle F, u_{1}-u_{0}\right\rangle,
$$

formula (3.1) can be written as

$$
\begin{aligned}
a_{1}\left(u_{1}-u_{0}, u_{1}-u_{0}\right)-\alpha\left(u_{0}, u_{0}\right) & =\delta W, \\
a_{0}\left(u_{1}-u_{0}, u_{1}-u_{0}\right)+\alpha\left(u_{1}, u_{1}\right) & =-\delta W, \\
\alpha\left(u_{0}, u_{1}\right) & =\delta W .
\end{aligned}
$$

We now prove estimates for $a$ and $\alpha$ that will be useful for our purposes. 
Proposition 3.3. Let $\lambda_{0}, \lambda_{1} \in(0,1]$ be given. Assume that $a_{0}$ and $a_{1}$ satisfy the following conditions:

$$
\begin{array}{ll}
\lambda_{0}\|u\|^{2} \leqslant a_{0}(u, u) \leqslant \lambda_{0}^{-1}\|u\|^{2} & \forall u \in H, \\
\lambda_{1}\|u\|^{2} \leqslant a_{1}(u, u) \leqslant \lambda_{1}^{-1}\|u\|^{2} & \forall u \in H .
\end{array}
$$

If $\alpha$ satisfies the condition

$$
0 \leqslant \alpha(u, u) \leqslant C_{0} a_{0}(u, u) \quad \forall u \in H,
$$

where $C_{0}$ is a positive constant, then

$$
|\delta W| \leqslant \alpha\left(u_{0}, u_{0}\right) \leqslant\left(1+C_{0}\right)|\delta W| .
$$

Conversely, if $\alpha$ satisfies the condition

$$
\alpha(u, u) \leqslant 0 \quad \forall u \in H
$$

then

$$
C|\delta W| \leqslant-\alpha\left(u_{0}, u_{0}\right) \leqslant|\delta W|,
$$

where $C$ is a positive constant depending only on $\lambda_{0}$ and $\lambda_{1}$.

Proof. Let us first consider (3.5). By $(3.3 b)$ we have $\delta W \leqslant 0$, and by $(3.3 a)$ we have $-\alpha\left(u_{0}, u_{0}\right) \leqslant \delta W$. Thus,

$$
|\delta W| \leqslant \alpha\left(u_{0}, u_{0}\right) .
$$

Let us now obtain the upper bound for $\alpha\left(u_{0}, u_{0}\right)$. Using lemma 3.2 we have

$$
\begin{aligned}
\alpha\left(u_{0}, u_{0}\right) \leqslant & \alpha\left(u_{0}-u_{1}, u_{0}-u_{1}\right)+\alpha\left(u_{1}, u_{1}\right) \\
& +2\left|\alpha\left(u_{0}-u_{1}, u_{0}-u_{1}\right)\right|^{1 / 2}\left|\alpha\left(u_{1}, u_{1}\right)\right|^{1 / 2} \\
\leqslant & \alpha\left(u_{0}-u_{1}, u_{0}-u_{1}\right)+\alpha\left(u_{1}, u_{1}\right) \\
& \quad+\varepsilon \alpha\left(u_{0}-u_{1}, u_{0}-u_{1}\right)+\frac{\alpha\left(u_{1}, u_{1}\right)}{\varepsilon} \\
\leqslant & (1+\varepsilon)\left[C_{0} a_{0}\left(u_{0}-u_{1}, u_{0}-u_{1}\right)+\frac{\alpha\left(u_{1}, u_{1}\right)}{\varepsilon}\right] \\
\leqslant & \left(1+C_{0}\right)|\delta W|
\end{aligned}
$$

where in the last line we have chosen $\varepsilon=1 / C_{0}$. Hence, we get

$$
\alpha\left(u_{0}, u_{0}\right) \leqslant\left(1+C_{0}\right)|\delta W| .
$$

Let us now consider (3.7). By $(3.3 a)$ we get $\delta W \geqslant 0$ and also

$$
\left|\alpha\left(u_{0}, u_{0}\right)\right| \leqslant \delta W .
$$

Let us recover an estimate from below for $\left|\alpha\left(u_{0}, u_{0}\right)\right|$. By $(3.3 c)$ we get

$$
\begin{aligned}
\delta W & =\alpha\left(u_{0}, u_{1}\right) \\
& \leqslant\left(-\alpha\left(u_{0}, u_{0}\right)\right)^{1 / 2}\left(-\alpha\left(u_{1}, u_{1}\right)\right)^{1 / 2} \\
& \leqslant \frac{\varepsilon}{2}\left(-\alpha\left(u_{1}, u_{1}\right)\right)+\frac{1}{2 \varepsilon}\left(-\alpha\left(u_{0}, u_{0}\right)\right) .
\end{aligned}
$$


Also, by $(3.3 b)$, we have

$$
-\alpha\left(u_{1}, u_{1}\right)=a_{0}\left(u_{1}-u_{0}, u_{1}-u_{0}\right)+\delta W
$$

Moreover, by $(3.4 a)$ and $(3.4 b)$, we have

$$
a_{0}\left(u_{1}-u_{0}, u_{1}-u_{0}\right) \leqslant \lambda_{0}^{-1} \lambda_{1}^{-1} a_{1}\left(u_{1}-u_{0}, u_{1}-u_{0}\right) .
$$

By the above inequality and (3.12) we obtain

$$
-\alpha\left(u_{1}, u_{1}\right) \leqslant \lambda_{0}^{-1} \lambda_{1}^{-1} a_{1}\left(u_{1}-u_{0}, u_{1}-u_{0}\right)+\delta W
$$

Then, substituting (3.11) and using (3.3a), we have

$$
\begin{aligned}
\delta W \leqslant & \frac{\varepsilon}{2}\left[A a_{1}\left(u_{1}-u_{0}, u_{1}-u_{0}\right)+\delta W\right]+\frac{1}{2 \varepsilon}\left(-\alpha\left(u_{0}, u_{0}\right)\right) \\
= & \frac{\varepsilon}{2}\left[A\left(a_{1}\left(u_{1}-u_{0}, u_{1}-u_{0}\right)-\alpha\left(u_{0}, u_{0}\right)\right)+A \alpha\left(u_{0}, u_{0}\right)+\delta W\right] \\
& \quad+\frac{1}{2 \varepsilon}\left(-\alpha\left(u_{0}, u_{0}\right)\right) \\
= & \frac{\varepsilon}{2}(1+A) \delta W+\left(\frac{1}{2 \varepsilon}-A \frac{\varepsilon}{2}\right)\left(-\alpha\left(u_{0}, u_{0}\right)\right)
\end{aligned}
$$

where $A:=\lambda_{0}^{-1} \lambda_{1}^{-1}$. Thus,

$$
\left(1-\frac{\varepsilon}{2}(1+A)\right) \delta W \leqslant \frac{1-A \varepsilon^{2}}{2 \varepsilon} \leqslant\left|\alpha\left(u_{0}, u_{0}\right)\right| .
$$

If $\varepsilon<\sqrt{A}$, we have

$$
\left(\frac{1}{1-A \varepsilon^{2}}\right)\left(2 \varepsilon\left(1-\frac{\varepsilon(1+A)}{2}\right)\right) \delta W \leqslant\left|\alpha\left(u_{0}, u_{0}\right)\right|
$$

Finally, choosing $\varepsilon=1 /(1+A)$, we get

$$
c \delta W \leqslant\left|\alpha\left(u_{0}, u_{0}\right)\right|
$$

where $c$ depends only on $\lambda_{0}$ and $\lambda_{1}$.

REMARK 3.4. In (3.5), condition (3.4) can be weakened by assuming $a_{0}(\cdot, \cdot)$ and $a_{1}(\cdot, \cdot)$ are positive semi-definite. Conversely, in $(3.7)$, it is enough to require that $a_{0}(\cdot, \cdot)$ and $a_{1}(\cdot, \cdot)$ are positive semi-definite, such that

$$
a_{0}(u, u) \leqslant C_{1} a_{1}(u, u) \quad \forall u \in H
$$

where $C_{1}$ is a positive constant. 


\section{Proof of the main result}

We want to make use of estimates obtained in the previous section to prove our bounds on the size of $E$. To do this we define

$$
\begin{aligned}
& a_{1}(u, v)=\int_{\Omega} \nabla u \cdot \nabla v \mathrm{~d} x+\int_{\Gamma} \gamma_{0} u v \mathrm{~d} \sigma+k \int_{E} u v \mathrm{~d} \sigma \\
& a_{0}(u, v)=\int_{\Omega} \nabla u \cdot \nabla v \mathrm{~d} x+\int_{\Gamma} \gamma_{0} u v \mathrm{~d} \sigma \\
& \alpha(u, v)=k \int_{E} u v \mathrm{~d} \sigma
\end{aligned}
$$

for $u, v \in H^{1}(\Omega)$. We immediately obtain

$$
\alpha(u, u)=k \int_{E} u^{2} \mathrm{~d} \sigma \geqslant 0, \quad a_{0}(u, u) \leqslant a_{1}(u, u),
$$

for every $u \in H^{1}(\Omega)$.

Lemma 4.1. There exists a constant $C>0$ depending only on $M$ and $L$ such that

$$
\int_{\partial \Omega \backslash \Gamma} u^{2} \mathrm{~d} \sigma \leqslant C\left(r_{0} \int_{\Omega}|\nabla u|^{2}+\int_{\Gamma_{0}} u^{2} \mathrm{~d} \sigma\right)
$$

for every $u \in H^{1}(\Omega)$.

Proof. By a standard trace inequality (see [1, ch. 7$]$ ) we get

$$
r_{0} \int_{\partial \Omega \backslash \Gamma} u^{2} \mathrm{~d} \sigma \leqslant C\left(\int_{\Omega}|u|^{2} \mathrm{~d} x+r_{0}^{2} \int_{\Omega}|\nabla u|^{2} \mathrm{~d} x\right) .
$$

Moreover, by the equivalence between the norm in $H^{1}(\Omega)$ and $\|\cdot\|_{*}$ introduced in remark 2.3, the thesis follows.

The main tools of unique continuation needed in the proof of our main result are contained in [21, lemma 4.5, theorem 4.6 and corollary 4.7], and for a detailed proof we refer the reader to [21]. However, for the reader's convenience and to make this paper as self-contained as possible, we sketch the proof of our main ingredient of unique continuation below.

Proposition 4.2 ( $A_{p}$ property on the boundary). Let $u_{0}$ be a solution to problem (1.3). Then there exist constants $p>1, A>0, \bar{r}>0$ depending only on the a priori data such that for every $x_{0} \in \Gamma_{1,2 \bar{r}}$ the following holds:

$$
\left(\frac{1}{\left|\Delta_{r}\left(x_{0}\right)\right|} \int_{\Delta_{r}\left(x_{0}\right)}\left|u_{0}\right|^{2}\right)\left(\frac{1}{\left|\Delta_{r}\left(x_{0}\right)\right|} \int_{\Delta_{r}\left(x_{0}\right)}\left|u_{0}\right|^{-2 /(p-1)}\right)^{p-1} \leqslant A,
$$

where $\Gamma_{1,2 \bar{r}}=\left\{x \in \partial \Omega\right.$ : $\left.\operatorname{dist}\left(x, \Gamma_{1}\right)<2 \bar{r}\right\}, \Gamma_{1}=\partial \Omega \backslash \bar{\Gamma}$ and $\Delta_{r}\left(x_{0}\right)=\Gamma_{1,2 \bar{r}} \cap B_{r}\left(x_{0}\right)$ with $0<r<\bar{r}$.

Proof. We recall that, as our main tool of unique continuation, the following socalled surface doubling inequality was achieved in [21]: there exists a constant 
$K_{1}>0$ depending only on the a priori data, such that, for any $x_{0} \in \Gamma_{1, \bar{r} / 2}$ and for every $r \in(0, \bar{r})$,

$$
\int_{\Delta_{2 r}\left(x_{0}\right)} u_{0}^{2} \leqslant K_{1} \int_{\Delta_{r}\left(x_{0}\right)} u_{0}^{2}
$$

holds. The proof of the latter has two main ingredients. The first is the well-known stability estimate for the Cauchy problem (see, for example, [22]):

$$
\begin{aligned}
& \int_{B_{r / 2}\left(x_{0}\right) \cap \Omega}\left|u_{0}\right|^{2} \leqslant C r\left(\int_{\Delta_{r}\left(x_{0}\right)} u_{0}^{2}+r^{2} \int_{\Delta_{r}\left(x_{0}\right)}\left|\nabla_{t} u_{0}\right|^{2}\right)^{1-\delta} \\
& \times\left(\int_{\Delta_{r}\left(x_{0}\right)} u_{0}^{2}+r^{2} \int_{\Delta_{r}\left(x_{0}\right)}\left|\nabla_{t} u_{0}\right|^{2}+\int_{B_{r}\left(x_{0}\right) \cap \Omega}\left|\nabla u_{0}\right|^{2}\right)^{\delta},
\end{aligned}
$$

where $\nabla_{t}$ denotes the tangential gradient on $\Delta_{r}\left(x_{0}\right)$ (more precisely, we have $\left.\nabla_{t} u_{0}=\nabla u_{0}-\left(\nabla u_{0} \cdot \nu\right)\right)$, and $C>0,0<\delta<1$ are constants depending only on the a priori data.

The second main ingredient is the following volume doubling inequality (see [21, lemma 4.5]):

$$
\int_{B_{\beta r}\left(x_{0}\right) \cap \Omega}\left|u_{0}\right|^{2} \leqslant C \beta^{K} \int_{B_{r}\left(x_{0}\right) \cap \Omega}\left|u_{0}\right|^{2}
$$

for every $r, \beta$ such that $\beta>1$ and $0<\beta r<2 \bar{r}$, where $C$ and $K$ are positive constants depending only on the a priori data. The inequality (4.7) was achieved in [21] by combining the techniques, introduced in [2], that apply to homogeneous Neumann boundary conditions, with a suitable change of variable that fits the problem under the assumption required in [2].

The control on the vanishing rate of the solution on the boundary provided by inequality (4.5) allowed Sincich [21, corollary 4.7] to obtain the following reverse Hölder inequality:

$$
\left(r^{-2} \int_{\Delta_{r}\left(x_{0}\right)} u_{0}^{2}\right)^{1 / 4} \leqslant\left(C^{-2} \int_{\Delta_{r}\left(x_{0}\right)} u_{0}^{2}\right)^{1 / 2},
$$

which in turn, combined with the powerful theory of Muckenhoupt weights (see [9]), leads to the desired integrability property for $\left|u_{0}\right|^{-1}$ in (4.4).

Proof of theorem 2.5. By lemma 4.1, there exists a positive constant $C_{1}$, depending on $M, L, c_{0}$, such that

$$
0 \leqslant \alpha(u, u) \leqslant C_{1} a_{0}(u, u) \quad \forall u \in H^{1}(\Omega) .
$$

By the above inequality and by proposition 3.3, we have

$$
|\delta W| \leqslant k \int_{E} u_{0}^{2} \mathrm{~d} \sigma \leqslant\left(1+C_{1}\right)|\delta W|
$$

where $\delta W=\int_{\partial \Omega} g u_{0}$. The leftmost inequality and standard bounds on the Neumann problem solution lead to the following inequality:

$$
|\delta W| \leqslant|E| k\left\|u_{0}\right\|_{L^{\infty}(E)}^{2} .
$$


Moreover, by a uniform boundedness-type estimate (see [14, ch. 8]) we have that

$$
|\delta W| \leqslant C \bar{k} r_{0}^{-1}|E|\left\|u_{0}\right\|_{H^{1}(\Omega)}^{2},
$$

where $C$ depends on the a priori data only. By remark 2.3 we also have that

$$
|\delta W| \leqslant C \bar{k}|E| r_{0}^{-n-1}\left(r_{0} \int_{\Gamma_{0}} u_{0}^{2}+r_{0}^{2} \int_{\Omega}\left|\nabla u_{0}\right|^{2}\right) .
$$

Moreover, by the lower bound in (2.3) we deduce that

$$
\begin{aligned}
|\delta W| & \leqslant C \bar{k}|E| r_{0}^{1-n} \max \left\{c_{0}^{-1}, 1\right\}\left(\int_{\Gamma_{0}} \gamma_{0} u_{0}^{2}+\int_{\Omega}\left|\nabla u_{0}\right|^{2}\right) \\
& \leqslant C \bar{k}|E| r_{0}^{1-n} \max \left\{c_{0}^{-1}, 1\right\}\left(\int_{\partial \Omega} \gamma u_{0}^{2}+\int_{\Omega}\left|\nabla u_{0}\right|^{2}\right) .
\end{aligned}
$$

Finally, by the weak formulation for $u_{0}$ (see remark 2.4) we have that

$$
|\delta W| \leqslant C \bar{k}|E| r_{0}^{1-n} \max \left\{c_{0}^{-1}, 1\right\}\left(\int_{\partial \Omega} g u_{0} \mathrm{~d} \sigma\right) .
$$

Let us consider now the upper bound for $E$. First, we have to cover properly the unknown part of the boundary (we refer the reader to [8] for a similar construction). Let $r$ be such that

$$
r=\frac{1}{4} \min \left\{\frac{r_{0}}{8 \sqrt{n}}, \frac{r_{0}}{2 \sqrt{n} M}\right\}
$$

and define

$$
\Gamma_{1}^{r}=\left\{x \in \Omega: \operatorname{dist}\left(x, \Gamma_{1}\right)<r\right\},
$$

where $\Gamma_{1}=\partial \Omega \backslash \bar{\Gamma}$.

Let $\left\{Q_{j}\right\}_{j=1}^{J}$ be a family of closed mutually internally disjoint cubes of size $2 r$ such that

$$
\begin{gathered}
\Gamma_{1}^{r} \cap Q_{j} \neq \emptyset, \quad j=1, \ldots, J, \\
\Gamma_{1}^{r} \subset \bigcup_{j=1}^{J} Q_{j} .
\end{gathered}
$$

Let $x_{j} \in \Gamma_{1}^{r} \cap Q_{j}, j=1, \ldots, J$. We have that

$$
\Gamma_{1}^{r} \subset \bigcup_{j=1}^{J} B_{4 \sqrt{n} r}\left(x_{j}\right) .
$$

Indeed, for $x \in \Gamma_{1}^{r}$, there exists $\bar{x} \in \Gamma_{1}$ such that $\operatorname{dist}(x, \bar{x})<2 \sqrt{n} r$. Let $j$ be such that $\bar{x} \in Q_{j}$. Since $\left|\bar{x}-x_{j}\right| \leqslant 2 \sqrt{n} r$, we have

$$
\left|x-x_{j}\right| \leqslant|x-\bar{x}|+\left|\bar{x}-x_{j}\right| \leqslant 4 \sqrt{n} r,
$$

which implies $x \in B_{4 \sqrt{n} r}\left(x_{j}\right)$. Making use of the construction argument in [8, proposition 5.2] we can infer that there exists a constant $C>0$ depending only on $M$ 
and $L$ such that

$$
\bigcup_{j=1}^{J} Q_{j} \subset\left\{x \in \mathbb{R}^{n}: \operatorname{dist}\left(x, \Gamma_{1}\right) \leqslant 4 \sqrt{n} r\right\} \text { and } J \leqslant C,
$$

where $C>0$ is a constant depending only on $M$ and $L$. By the Hölder inequality, (3.6) and (4.3) we have

$$
\begin{aligned}
|E| & =\int_{E}\left|u_{0}\right|^{-2 / p}\left|u_{0}\right|^{2 / p} \\
& \leqslant\left(\int_{E}\left|u_{0}\right|^{-2 /(p-1)}\right)^{(p-1) / p}\left(\int_{E}\left|u_{0}\right|^{2}\right)^{1 / p} \\
& \leqslant\left(\int_{\Gamma_{1}}\left|u_{0}\right|^{-2 /(p-1)}\right)^{(p-1) / p}\left(\left(1+C_{0}\right)|\delta W|\right)^{1 / p},
\end{aligned}
$$

where $C$ depends only on $M, L$ and $c_{0}$. Now,

$$
\begin{aligned}
\int_{\Gamma_{1}}\left|u_{0}\right|^{-2 /(p-1)} & \leqslant \int_{\Gamma_{1} \cap\left(\bigcup_{j=1}^{J} B_{4 \sqrt{n} r}\left(x_{j}\right)\right)}\left|u_{0}\right|^{-2 /(p-1)} \\
& \leqslant \sum_{j=1}^{J} \int_{\Delta_{j}}\left|u_{0}\right|^{-2 /(p-1)} \\
& \leqslant \sum_{j=1}^{J} \frac{L r_{0}^{n-1}}{\left|\Delta_{j}\right|} \int_{\Delta_{j}}\left|u_{0}\right|^{-2 /(p-1)}
\end{aligned}
$$

where $\Delta_{j}=B_{4 \sqrt{n} r}\left(x_{j}\right) \cap \Gamma_{1}$. By proposition 4.2 , we have that

$$
\frac{1}{\left|\Delta_{j}\right|} \int_{\Delta_{j}}\left|u_{0}\right|^{-2 /(p-1)} \leqslant\left(\frac{A}{\left(1 /\left|\Delta_{j}\right|\right) \int_{\Delta_{j}}\left|u_{0}\right|^{2}}\right)^{1 /(p-1)},
$$

where $A$ is a constant depending only on $M, L, c_{0}$ and $F$. Let us assume that the index $\bar{j}, 1 \leqslant \bar{j} \leqslant J$, is such that

$$
\frac{1}{\left|\Delta_{\bar{j}}\right|} \int_{\Delta_{\bar{j}}}\left|u_{0}\right|^{2}=\min _{1 \leqslant j \leqslant J} \frac{1}{\left|\Delta_{j}\right|} \int_{\Delta_{j}}\left|u_{0}\right|^{2} .
$$

By combining (4.15), (4.16) and (4.18), we have that

$$
|E| \leqslant\left(J \operatorname{Lr}_{0}^{n-1}\left(\frac{A}{\left(1 /\left|\Delta_{\bar{j}}\right|\right) \int_{\Delta_{\bar{j}}}\left|u_{0}\right|^{2}}\right)^{1 /(p-1)}\right)^{(p-1) / p}\left(\left(1+C_{0}\right)|\delta W|\right)^{1 / p} .
$$

By the a priori bound $|\partial \Omega| \leqslant L r_{0}^{n-1}$, we easily get that

$$
\frac{1}{\left|\Delta_{\bar{j}}\right|} \int_{\Delta_{\bar{j}}}\left|u_{0}\right|^{2} \geqslant \frac{1}{L r_{0}^{n-1}} \int_{\Delta_{\bar{j}}}\left|u_{0}\right|^{2} .
$$

By (4.5) and by a standard trace inequality we can infer that

$$
\int_{\Delta_{\bar{j}}\left(x_{j}\right)}\left|u_{0}\right|^{2} \geqslant C r_{0}^{-1} \int_{B_{2 \sqrt{n} r}\left(x_{j}\right) \cap \Omega}\left|u_{0}\right|^{2},
$$


where $C>0$ is a constant depending only on $\bar{k}_{0}, \bar{k}_{1}, M, L$ and $F$. Let $\bar{x} \in$ $B_{2 \sqrt{n} r}\left(x_{j}\right) \cap \Omega$ be such that $B_{\sqrt{n} r / 4}(\bar{x}) \subset B_{2 \sqrt{n} r}\left(x_{j}\right) \cap \Omega$. Hence, we get

$$
\int_{\Delta_{\bar{j}}\left(x_{j}\right)}\left|u_{0}\right|^{2} \geqslant C r_{0}^{-1} \int_{B_{\sqrt{n} r / 4}(\bar{x})}\left|u_{0}\right|^{2} .
$$

Now, using the arguments developed in [18, proposition 3.1] (see also [7, lemma 5.3]), relying on a standard propagation of smallness, we get

$$
\int_{B_{\sqrt{n} r / 4}(\bar{x})}\left|u_{0}\right|^{2} \geqslant C \int_{\Omega}\left|u_{0}\right|^{2},
$$

where $C>0$ is a constant depending only on $M, L, \bar{k}_{0}, \bar{k}_{1}$ and $F$. Hence, combining (4.20), (4.22) and (4.23), it easily follows that

$$
\frac{1}{\left|\Delta_{\bar{j}}\right|} \int_{\Delta_{\bar{j}}}\left|u_{0}\right|^{2} \geqslant C r_{0}^{-n} \int_{\Omega}\left|u_{0}\right|^{2},
$$

where $C>0$ is a constant depending only on $M, L, \bar{k}_{0}, \bar{k}_{1}$ and $F$. By the estimate (4.22) and the Caccioppoli inequality we get that

$$
\int_{\Delta_{\bar{j}}}\left|u_{0}\right|^{2} \geqslant C r_{0} \int_{B_{\sqrt{n} r / 8}(\bar{x})}\left|\nabla u_{0}\right|^{2},
$$

where $C>0$ is a constant depending only on $M, L, \bar{k}_{0}, \bar{k}_{1}$ and $F$. Repeating the propagation of smallness techniques described in [18, proposition 3.1], this time for the gradient, we get that

$$
\frac{1}{\left|\Delta_{\bar{j}}\right|} \int_{\Delta_{\bar{j}}}\left|u_{0}\right|^{2} \geqslant C r_{0}^{2-n} \int_{\Omega}\left|\nabla u_{0}\right|^{2},
$$

where $C>0$ is a constant depending only on $M, L, \bar{k}_{0}, \bar{k}_{1}$ and $F$. We can then infer that

$$
\frac{1}{\left|\Delta_{\bar{j}}\right|} \int_{\Delta_{\bar{j}}}\left|u_{0}\right|^{2} \geqslant C\left(r_{0}^{-n} \int_{\Omega}\left|u_{0}\right|^{2}+r_{0}^{2-n} \int_{\Omega}\left|\nabla u_{0}\right|^{2}\right)
$$

where $C>0$ is a constant depending only on $M, L, \bar{k}_{0}, \bar{k}_{1}$ and $F$. By the equivalence between the standard $H^{1}(\Omega)$ norm and the norm introduced in remark 2.3 we find that

$$
\frac{1}{\left|\Delta_{\bar{j}}\right|} \int_{\Delta_{\bar{j}}}\left|u_{0}\right|^{2} \geqslant C r_{0}^{-n}\left(r_{0} \int_{\Gamma_{0}}\left|u_{0}\right|^{2}+r_{0}^{2} \int_{\Omega}\left|\nabla u_{0}\right|^{2}\right)
$$

where $C>0$ is a constant depending only on $M, L, \bar{k}_{0}, \bar{k}_{1}$ and $F$. Now, by the a priori bound $\gamma_{0}(x) \leqslant c_{0}^{-1} / r_{0}$ on $\Gamma$, we get that

$$
\begin{aligned}
\frac{1}{\left|\Delta_{\bar{j}}\right|} \int_{\Delta_{\bar{j}}}\left|u_{0}\right|^{2} & \geqslant C r_{0}^{2-n} \min \left\{1, c_{0}\right\}\left(\int_{\Gamma_{0}} \gamma_{0}\left|u_{0}\right|^{2}+\int_{\Omega}\left|\nabla u_{0}\right|^{2}\right) \\
& \geqslant C r_{0}^{2-n} \min \left\{1, c_{0}\right\} \int_{\partial \Omega} g u_{0} .
\end{aligned}
$$


Combining (4.14), (4.19) and (4.29) and recalling that $\int_{\partial \Omega} g u_{0}=W_{0}$, we obtain that

$$
|E| \leqslant C r_{0}^{n-1}\left(\frac{W-W_{0}}{W_{0}}\right)^{1 / p},
$$

where $C>0$ is a constant depending only on $M, L, \bar{k}_{0}, \bar{k}_{1}, F$ and $c_{0}$.

\section{References}

1 R. A. Adams. Sobolev spaces (New York: Academic Press, 1975).

2 V. Adolfsson and L. Escauriaza. $C^{1, \alpha}$ domains and unique continuation at the boundary. Commun. Pure Appl. Math. 50 (1997), 935-969.

3 G. Alessandrini and E. Rosset. The inverse conductivity problem with one measurement: bounds on the size of the unknown object. SIAM J. Appl. Math. 58 (1998), 1060-1071.

4 G. Alessandrini, E. Rosset and J. K. Seo. Optimal size estimates for the inverse conductivity problem with one measurement. Proc. Am. Math. Soc. 128 (2000), 53-64.

5 G. Alessandrini, A. Morassi and E. Rosset. Size estimates. In Inverse problems: theory and applications. Contemporary Mathematics, vol. 333, pp. 1-33 (Providence, RI: American Mathematical Society, 2003).

6 G. Alessandrini, A. Morassi and E. Rosset. The linear constraints in Poincaré and Korn type inequalities. Forum Math. 28 (2008), 557-569.

7 G. Alessandrini, E. Sincich and S. Vessella. Stable determination of surface impedance on a rough obstacle by far field data. Inv. Probl. Imaging 7 (2013), 341-351.

8 E. Beretta, E. Francini and S. Vessella. Size estimates for the EIT problem with one measurement: the complex case. Rev. Mat. Ibero. 30 (2014), 551-580.

9 R. R. Coifman and C. L. Fefferman. Weighted norm inequalities for maximal function and singular integrals. Studia Math. 51 (1974), 241-250.

10 M. Di Cristo, C-L. Lin, A. Morassi, E. Rosset, S. Vessella and J.-N. Wang. Doubling inequalities for anisotropic plate equations and applications to size estimates of inclusions. Inv. Probl. 29 (2013), 125012.

11 M. Di Cristo, C.-L. Lin, S. Vessella and J.-N. Wang. Detecting a general inclusion in the shallow shell. SIAM J. Math. Analysis 45 (2013), 88-100.

12 M. Di Cristo, C.-L. Lin and J.-N. Wang. Quantitative uniqueness estimates for the shallow shell system and their applications to an inverse problem. Annali Scuola Norm. Sup. Pisa IV 12 (2013), 43-92.

13 A. Friedman. Detection of mines by electric measurements. SIAM J. Appl. Math. 47 (1987), 201-212.

14 D. Gilbarg and N. S. Trudinger. Elliptic partial differential equations of second order, 2nd edn, Classics in Mathematics (Springer, 2001).

15 H. Kang, J. K. Seo and D. Sheen. The inverse conductivity problem with one measurement: stability and estimation of size. SIAM J. Math. Analysis 28 (1997), 1389-1405.

16 P. G. Kaup, F. Santosa and M. Vogelius. A method for imaging corrosion in thin plates from electrostatic data. Inv. Probl. 12 (1996), 279-93.

17 J. J. Lions and E. Magenes. Non-homogeneous boundary value problems and applications, vol. 1 (English transl.). Die Grundlehren der mathematischen Wissenschaften, vol. 181 (Springer, 1972).

18 A. Morassi and E. Rosset. Stable determination of cavities in elastic bodies. Inv. Probl. 20 (2004), 453-480.

19 A. Morassi, E. Rosset and S. Vessella. Size estimates for inclusions in an elastic plate by boundary measurements. Indiana Univ. Math. J. 56 (2007), 2325-2384.

20 A. Morassi, E. Rosset and S. Vessella. Estimating area of inclusions in anisotropic plates from boundary data. Discrete Contin. Dynam. Syst. 6 (2013), 501-515.

21 E. Sincich. Stable determination of the surface impedance of an obstacle by far field measurements. SIAM J. Math. Analysis 38 (2006), 434-451.

22 G. N. Trytten. Pointwise bound for solution of the Cauchy problem for elliptic equations. Arch. Ration. Mech. Analysis 13 (1963), 222-224.

23 M. Vogelius and J.-M. Xu. A nonlinear elliptic boundary value problem related to corrosion modeling. Q. Appl. Math. 56 (1998), 479-505. 